\title{
Transcranial stimulation in semantic dementia
}

\author{
Elsa Mhanna ${ }^{1}$, Clara Sanches ${ }^{1}$, Antoni Valero-Cabré ${ }^{1}$, Marc Teichmann ${ }^{1,2}$ \\ ${ }^{1}$ Institut du Cerveau et la Moelle Épinière, Sorbonne Université, France. \\ ${ }^{2}$ Department of Neurology, Assistance Publique Hôpitaux de Paris, France. \\ https://doi.org/10.36505/ExLing-2019/10/0036/000398
}

\begin{abstract}
Semantic variant of primary progressive aphasia (sv-PPA) is the most frequent form of PPA to with no current treatment. Non-invasive brain stimulation by transcranial Direct Current Stimulation (tDCS) is emerging as a therapeutic alternative. We present preliminary results from a double-blind sham-controlled study of $14 \mathrm{sv}$-PPA patients who received daily tDCS sessions for 10 consecutive days. Patients were randomized to 3 conditions: Left Anodal (excitatory), Right Cathodal (inhibitory) and Sham (Placebo). Participants underwent series of language tasks, prior and following the sessions. Prior and 2 weeks following the tDCS regime patients underwent ${ }^{18}[\mathrm{~F}]-F D G-P E T$ recordings. We didn't find significant improvements in semantic access for any group. Left Anodal group showed medium effect sizes in visual sematic associations. Excellent tolerance and a high level of subjective satisfaction was found for all groups. The protocol is ongoing.
\end{abstract}

Key words: Semantic variant, primary progressive aphasia, repetitive tDCS

\section{Introduction}

The semantic variant of primary progressive aphasia (sv-PPA) or Semantic Dementia is the most frequent of all PPA forms and its main feature is a loss of conceptual knowledge with preserved fluency and repetition (Gorno-Tempini 2004). Little can currently be done to effectively treat sv-PPA patients.

Transcranial direct current stimulation (tDCS) has been employed in neurodegenerative diseases with encouraging results (Cotelli 2014). This technology delivers a weak (1-2 mA) constant current between two electrodes (an anode and a cathode) placed on separate scalp locations.

Results from a pre-therapeutic study (Teichmann 2016) evaluated the impact of a single tDCS session on a cohort of $12 \mathrm{sv}$-PPA patients. The current protocol is a prospective double-blind randomized clinical trial evaluating the impact of a periodical regime of daily tDCS sessions. We compared the impact of 2 stimulation strategies: Anodal (excitatory) tDCS on the left ATL (Anterior temporal lobe) to boost impaired systems of the left hemisphere involved in semantic access and, Cathodal (inhibitory) tDCS, targeting the right ATL, to suppress interhemispheric inhibitory interactions and a Sham (placebo).

ExLing 2019: Proceedings of $10^{\text {th }}$ International Conference of Experimental Linguistics, 25-27 September 2019, Lisbon, Portugal 


\section{Material and methods}

14 patients diagnosed with sv-PPA were enrolled in our study at the PitieSalpêtrière University Hospital. Diagnosis of sv-PPA was made according to international criteria (Gorno-Tempini et al., 2011).

A cohort of healthy controls $(\mathrm{HC}, \mathrm{n}=5)$ were matched with our patients for gender, age, socio-cultural level and handedness (differences for all matched variables between sv-PPA and HC $\mathrm{p}>0.05)$. Patients were randomized into 3 groups. All gave informed signed consent.

Table 1: Demographical information about the participants in the study.

\begin{tabular}{lllll}
\hline \hline Tests & LA tDCS & RC tDCS & Sham tDCS & HC \\
\hline & & & & \\
Nr of Participants & 5 & 4 & 5 & 5 \\
Sex M/F & $3 / 2$ & $3 / 1$ & $3 / 2$ & $4 / 1$ \\
Age (years) & $68,6( \pm 5,3)$ & $66,7( \pm 5,1)$ & $73,4( \pm 2,1)$ & $64,6( \pm 6,9)$ \\
$\begin{array}{l}\text { Disease duration } \\
\text { (years) }\end{array}$ & $5( \pm 1,4)$ & $3,7( \pm 0,7)$ & $4( \pm 1,7)$ & - \\
\hline \hline
\end{tabular}

LA: Left anodal; RC: Right Cathodal; HC: Healthy Controls.

This clinical trial is a double-blind sham-controlled study involving 10 daily tDCS sessions over 2 weeks. All behavioral evaluations were performed at 4 time-points of the clinical follow up: pre tDCS baseline, 3 days after the $10^{\text {th }}$ and final tDCS session, 2 weeks later and 4 months later.. Healthy controls were only evaluated once following identical behavioral tasks and neuroimaging protocols as sv-PPA patients. To deliver tDCS we used a device by NE STARSTIM' model 'ENOBIO' 8 channels. During active anodal or cathodal stimulation (LA and RC groups), current was leveled up to reach an intensity $1.59 \mathrm{~mA}$ (current density: $0.06 \mathrm{~mA} / \mathrm{cm}^{2}$ ) (Teichmann 2016). Stimulation lasted 20 minutes.

A verbal and visual semantic association tasks that include 78 words (SA verbal) or pictures (SA visual) grouped in 26 trials each assembling 3 words or pictures in which only 2 are related semantically.

All patients underwent ${ }^{18}[\mathrm{~F}]$-FDG-PET $\left({ }^{18}[\mathrm{~F}]\right.$-Fluoro-2-Desoxy-2-Fluoro-DGlucose Positron Emission Tomography) at baseline and 2 weeks after the end of the 10 days tDCS treatment to document and quantify lasting modifications of temporal lobe metabolism by the stimulation regime.

\section{Results}

The three groups of sv-PPA patients achieved significantly lower accuracy levels than their matched healthy controls (HC) at inclusion. A non-parametric Wilcoxon test applied to each comparison yielded statistically significant results 
$(\mathrm{p}<0.05)$ for all three comparisons (LA vs HC, RC vs HC and sham vs HC) (See Figure 1).
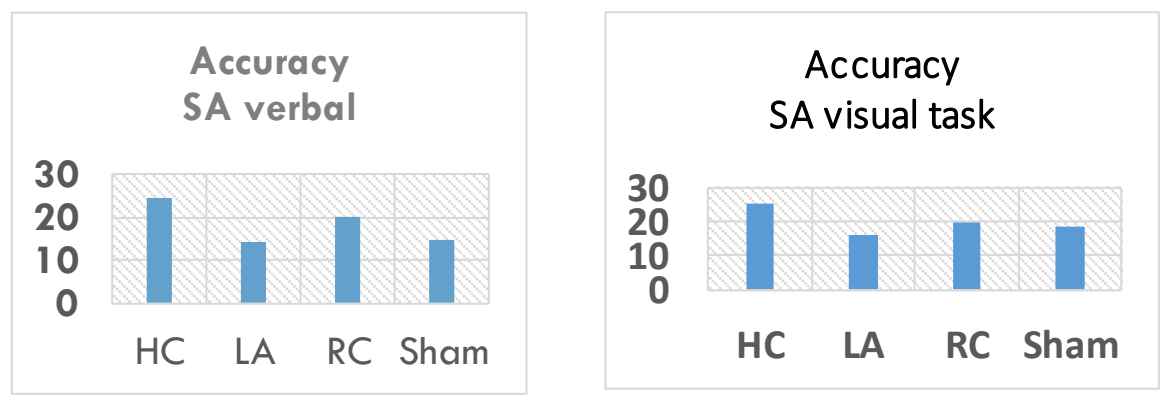

Figure 1. Mean accuracy for the verbal Semantic Association (SA verbal) and visual Semantic Association (SA visual) tasks measured at inclusion in healthy controls $(\mathrm{HC}, \mathrm{n}=5)$ and the sv-PPA patients randomized to Left Anodal tDCS $(\mathrm{LA}, \mathrm{n}=5)$, Right Cathodal tDCS $(\mathrm{RC}, \mathrm{n}=4)$ or Sham tDCS (Sham, $\mathrm{n}=5$ ) groups.
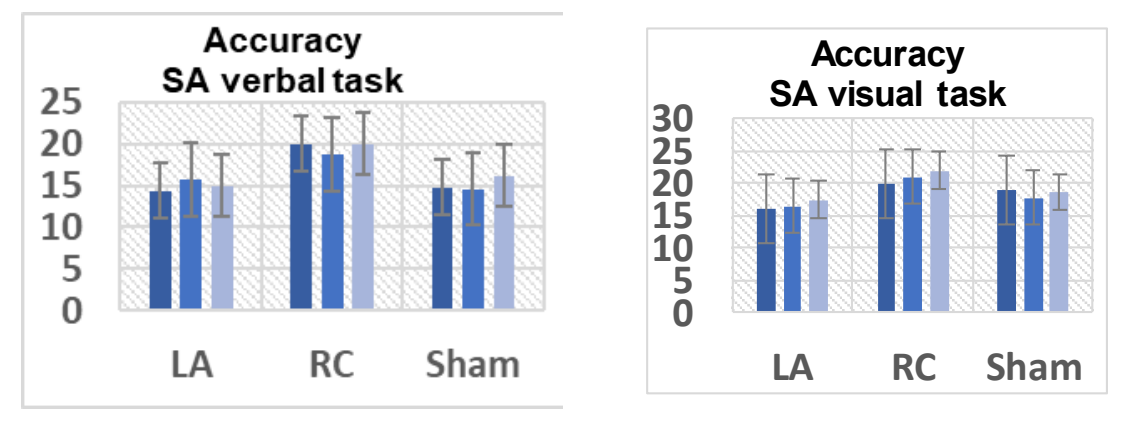

Figure 2. Preliminary outcomes calculated for three time-points across our follow up for Semantic association tasks. Data are presented for Baseline, 3 days post-stimulation (Post 1) and 2 weeks after tDCS offset (Post 2) and separated for each tDCS group: Left Anodal tDCS (LA), Right Cathodal tDCS (RC) and Sham tDCS groups. Time points for each tDCS group (LA, RC and SHAM) are presented serially in blue hues (from dark to light blue, Baseline, Post 1 and Post 2) in correlative order.

We performed non-parametric paired and unpaired tests to identify potential effects of tDCS stimulation based on the tasks. We compared post vs. pre tDCS regime accuracy shifts (Wilcoxon rank test) for each individual stimulation groups. We also explored significant differences between post minus pre accuracy differences, comparing active tDCS (LA, RC) with sham tDCS (SHAM) groups (U-Mann Withney test). It yielded no significant results for any tDCS groups (All Wilcoxon rank tests $\mathrm{p}>0.05$ ). Similarly, comparison of post 
148 E. Mhanna et al.

minus pre tDCS accuracy differences across groups did not reveal any statistically significant difference (All U-Mann-Withney tests $\mathrm{p}>0.05$ ). See Figure 2. Potentially interesting, a Cohen's d test showed a mild effect size for Left Anodal tDCS stimulation $(\mathrm{d} 0,46)$ in the SA visual task compared with the other tDCS groups (RC and SHAM).

Finally, we attempted to identify a metabolic ${ }^{18}[\mathrm{~F}]$-FDG-PET biomarker at inclusion able to predict improvements driven by active tDCS treatment. One significant correlation was noted between metabolism in the right middle temporal pole and SA visual task improvements in both active tDCS groups ( $\mathrm{p}$ value of 0.04, r 0,69 Pearson correlation).

\section{Discussion and conclusion}

Thus far, we can conclude that our 10 days tDCS regime showed to be well tolerated and no stimulation negative side-effects were noted in concordance with our pilot study (Teichmann 2016). Even if very preliminary, correlation between the semantic tasks and the ${ }^{18}[$ F]-FDG-PET metabolic levels showed a statistically significant correlation between FDG metabolism in the right middle temporal pole and post-pre tDCS outcomes for the SA visual task. Such observation could suggest a potential role for the right hemisphere subtending in visual semantic processes. On the other hand, we were unable yet to find clear evidence supporting the beneficial effects of active tDCS stimulation on the language tasks. However, the medium effect size found for the left anodal tDCS group (LA) compared to the other two (RC and Sham) suggests that with a higher number of patients statistically significant differences might arise between active tDCS and sham groups.

\section{References}

Gorno-Tempini M.L., Dronkers N.F., Rankin K.P., Ogar, J.M., Phengramy, L., Rosen, H.J. Johnson, J.K. Weiner, M.W., Miller, B.L. 2004. Cognition and anatomy in three variants of primary progressive aphasia. Ann Neurol 2004, 55, 335-346.

Cotelli, M., Manenti, R., Petesi, M., Brambilla, M., Cosseddu, M. Zanett, O. Miniussi, C., Padovani, A., Borroni, B. 2014. Treatment of Primary Progressive Aphasias by Transcranial Direct Current Stimulation Combined with Language Training. Journal of Alzheimer's Disease 39, 799-808.

Teichmann M., Lesoil C., Godard J., Vernet, M., Bertrand, A., Levy, R. Dubois, B., Lemoine, L., Truong D.Q., Bikson, M., Kas, A., Valero-Cabre, A. 2016. Direct current stimulation of temporal poles boosts semantic 93-707. Processing in primary progressive aphasia. Annals of Neurology 2016, 80 (5): 6.

Valero-Cabré, A., Sanches, C., Godard, J., Fracchia, O., Dubois, B., Levy, R., Truong, D.Q., Bikson, M., Teichmann, M. 2019. Language boosting by transcranial stimulation in Progressive Supranuclear Palsy. Neurology 2019. 